\title{
Avaliação postural de crianças com mielomeningocele: um estudo de revisão
}

\author{
Postural evaluation of children with myelomeningocele: a review study \\ Evaluación postural de niños con mielomeningocele: \\ un estudio de revisión \\ Thaynara do Monte MÉLO' \\ Paulo Henrique Meira DUARTE1 \\ Hellen Cristina Barbosa PEREIRA1 \\ Natália de Fátima Meireles PEREIRA 1 \\ Rielly Maria Cruz da SILVA2 \\ Nícia Farias Braga MACIEL ${ }^{3}$
}

\author{
${ }^{1}$ Fisioterapeutas pela Faculdade Maurício de Nassau, UNINASSAU, 58030-000, João Pessoa - PB, Brasil \\ ${ }^{2}$ Graduanda do curso de Fisioterapia da Faculdade Maurício de Nassau, UNINASSAU, 58030-000, João Pessoa - PB, Brasil \\ ${ }^{3}$ Docente Mestre do curso de Fisioterapia da Faculdade Maurício de Nassau, UNINASSAU, 58030-000, João Pessoa - PB, Brasil
}

\begin{abstract}
Resumo
Introdução: A mielomeningocele também conhecida como disrafismo espinhal ou espinha bífida aberta, é um tipo de anomalia congênita do sistema nervoso central, que provoca graves complicações neurológicas e compromete o estado da qualidade de vida da criança. Objetivo: Realizar uma revisão sistemática acerca da avaliação postural de crianças com mielomeningocele. Material e método: O planejamento para esta revisão sistemática, de categoria específica, foi constituído por artigos ancorados ao setor de saúde e publicados entre os anos de 2007 e 2017. A Biblioteca Virtual em Saúde foi empregue para realizar a coleta das informações acerca da temática e os descritores definidos para a pesquisa foram: mielomeningocele, disrafismo espinhal, postura, fisioterapia e crianças. Resultados: Foi localizada uma soma de 346 estudos com o uso dos descritores já mencionados. Consecutivo a todas as exigências aplicadas, apenas sete artigos foram eleitos para produzir esta revisão. Os dados encontrados são característicos de uma população infantil com perfil de MMC e correspondem a bons resultados por meio do método de avaliação postural, que se fundamenta na inspeção minuciosa das limitações posturais resultantes de irregularidades no corpo. Em vista disso, a maioria dos estudos apresentou o pé torto congênito como traço dominante nos pacientes portadores. Conclusão: Diante dos informes obtidos, foi possível constatar que a avaliação postural em crianças com mielomeningocele pode gerar resultados satisfatórios para o meio acadêmico e para os demais profissionais da área de saúde, de modo especial ao fisioterapeuta, que além de identificar os desníveis existentes no alinhamento corporal, também é capaz de intervir recuperando a mobilidade funcional deficitária dos pacientes.
\end{abstract}

Descritores: Mielomeningocele; Disrafismo Espinhal; Postura; Fisioterapia; Criança.

\section{Abstract}

Introduction: Myelomeningocele also as known as spinal dysraphism or open spina bifida, is a type of congenital anomaly of the central nervous system, which to provoke severe neurological complications and compromises the state of the child's quality of life. Objective: To achieve a systematic review about the postural evaluation of children with myelomeningocele. Material and method: The planning for systematic review, of specific category, was constituted by articles anchored to the health sector and published between the years of 2007 and 2017. The Virtual Health Library, was used to realize information collection about the theme and the descriptors defined for the research were: myelomeningocele, spinal dysraphism, posture, physiotherapy and children. Results: Was found a sum of three hundred and forty six studies using the descriptors already mentioned. Consistent with all applied requirements, only seven articles were elected to produce this review. The data found are characteristic of a child population with a myelomeningocele profile and correspond to good results through the method of postural evaluation, which is based on the complete inspection of the postural limitation resulting from irregularities in the body. In view of this, the majority of the studies presented the congenital crooked foot as the dominant trait in the patients. Conclusion: In view of the obtained reports, it was possible to verify that the postural evaluation in children with myelomeningocele can generate satisfactory results for the academic environment and for the other professionals of the health area, in a special way to the physiotherapist, who in addition to identifying existing gaps body alignment, it also able to intervene by recovering the deficient functional mobility of the patients.

Descriptors: Myelomeningocele; Spinal Dysraphism; Posture; Physiotherapy; Children.

\section{Resumen}

Introducción: La mielomeningocele también conocida como disrafismo espinal o espina bífida abierta, es un tipo de anomalía congénita del sistema nervioso central, que provoca graves complicaciones neurológicas y compromete el estado de la calidad de vida del niño. Objetivo: Realizar una revisión sistemática sobre la evaluación postural de niños con mielomeningocele. Material y método: La planificación para esta revisión sistemática, de categoría específica, fue constituida por artículos anclados al sector de salud y publicados entre los años 2007 y 2017. La Biblioteca Virtual en Salud fue empleada para realizar la recolección de las informaciones acerca de la temática y los descriptores definidos para la investigación fueron: mielomeningocele, disrafismo espinal, postura, fisioterapia y niños. Los resultados: Se ha localizado una suma de 346 estudios con el uso de los descriptores ya mencionados. Consecutivo a todas las exigencias aplicadas, sólo siete artículos fueron elegidos para producir esta revisión. Los datos encontrados son característicos de una población infantiles con perfiles de mielomeningocele y corresponden a buenos resultados por medio del método de evaluación postural, que se fundamenta en la inspección minuciosa de las limitaciones posturales resultantes de irregularidades en el cuerpo. En vista de ello, la mayoría de los estudios presentó el pie torcido congénito como rasgo dominante en los pacientes portadores. Conclusión: Ante los informes obtenidos, fue posible constatar que la evaluación postural en niños con mielomeningocele puede generar resultados satisfactorios para el medio académico y para los demás profesionales del área de salud, de modo especial al fisioterapeuta, que además de identificar los desniveles existentes en la alineación corporal, también es capaz de intervenir recuperando la movilidad funcional deficitaria de los pacientes.

Descriptores: Mielomeningocele; Disrafia Espinal; Postura; Fisioterapia; Niños.

\section{INTRODUÇÃO}

A mielomeningocele (MMC) também conhecida como disrafismo espinhal ou espinha bífida aberta, é um tipo de anomalia congênita do sistema nervoso central (SNC), que provoca graves complicações neurológicas e compromete o estado da qualidade de vida da criança. Sobrevém de uma alteração no tubo neural considerada como um defeito decorrente de uma falha no desenvolvimento do fechamento da estrutura, passando a ser detectada por meio do diagnóstico intrauterino ${ }^{1}$

A MMC é compreendida como uma falha severa que impede o fechamento do tubo neural e origina diversas complicações, cuja característica remete à exteriorização da estrutura e exposição de tecido nervoso através de uma bolsa cística (Figura 1). O defeito congênito é capaz de complicar a 
saúde da criança com diversas consequências, sobretudo com as mais comuns, como a paralisia, as alterações de sensibilidade no tecido epitelial de revestimento, o déficit para autocontrole de bexiga e as deformidades musculares ${ }^{2}$.

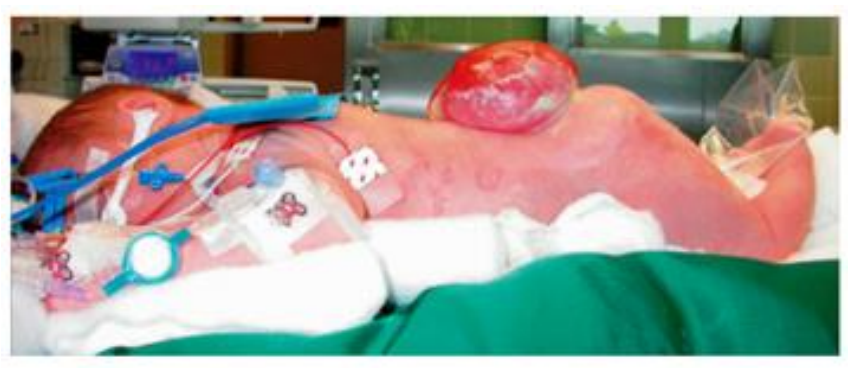

Figura 1. Malformação de uma espinha bífida aberta: mielomeningocele (MMC) de acordo com Biedermann ${ }^{13}$

A causa dessa patologia não é detalhada com especificidade, porém, a análise etiológica da doença apresenta evidências multifatoriais, abrangendo condições ambientais, fatores genéticos, circunstâncias orgânicas e até mesmo nutricionais, tendo como exemplo para este último o ácido fólico, considerado pela literatura como causador de risco $^{3}$.

Os elementos epidemiológicos apresentam uma carência de dados referentes às taxas de incidência das deformidades do tubo neural, principalmente da MMC que retrata a condição mais severa da espinha bífida. A estatística mais atual disponibiliza resultados que revelam taxas variantes de $0,83 / 1.000$ a $1,87 / 1.000$ nascimentos ${ }^{4}$.

As manifestações clínicas resultam em um mau funcionamento de órgãos e sistemas, atingindo os ossos, a epiderme, as vias dos tratos responsáveis pelo aparelho digestivo e urinário, assim como, o SNC e periférico. Quanto a classificação, a MMC pode estar presente em qualquer extensão da coluna com exposição da medula espinhal, sendo a área lombossacral responsável pelo nível de comprometimento medular mais comum, correspondendo a cerca de $75 \%$ dos casos. É importante ressaltar que a dimensão e o estado da função neurológica dependem do local em que a malformação é encontrada, bem como às lesões secundárias ${ }^{3}$.

Os diferentes níveis de lesões indicam as possíveis alterações no grau de motricidade de cada criança. Quanto maior for a altura da lesão, pior será o prognóstico para se alcançar a marcha. A lesão medular pode ocorrer em diferentes pontos da coluna vertebral, estando comumente ligada a problemas de interação social e mobilidade, classificados como danos que prejudicam a função motora da região torácica, lombar alta, lombar baixa e sacral ${ }^{1}$.

O comprometimento medular é um fator contribuinte para determinar o nível neurológico da lesão relativo à capacidade de deambulação. Crianças com níveis de lesão baixa indicam maior funcionalidade, porém, não estão dispensadas de problemas ortopédicos, úlceras de pressão, insuficiência renal e déficits na capacidade de micção, evacuação e movimentação. Há também outros motivos relevantes que influenciam e prejudicam o potencial deambulatório, como, por exemplo, as contraturas e deformidades localizadas na coluna vertebral e nos membros inferiores, que interferem de forma direta o processo de deambulação 5 .

Dentre as disfunções encontradas na MMC, as alterações posturais são observadas com seriedade. Sendo ocasionadas por mudanças no alinhamento biomecânico, apresentam algumas complicações ortopédicas como o realce da curvatura cifótica, contraturas, subluxação congênita de quadril, deformidades evidentes na região do joelho e tornozelo, pé torto e também algumas características clínicas como paralisia e fraqueza neuromuscular nas extremidades inferiores, bexiga neurogênica, hidrocefalia e interferência na função do aparelho gastrointestinal ${ }^{3}$.

A análise da postura abrange uma relevante importância e interesse sobre a biomecânica e funcionalidade do corpo humano, na qual inspeciona e relaciona as alterações do alinhamento esquelético com o nível de lesão, em razão do atraso no desenvolvimento neuropsicomotor (DNPM) que a patologia provoca sobre a criança. Tendo em conta que os desajustes posturais requerem uma assistência global, o fisioterapeuta dispõe plena responsabilidade e competência para atuar com maior relevância no segundo nível de atenção à saúde, prevenindo futuras deformidades por meio da cinesioterapia, com a prática de exercícios passivos, ativos e de resistência, as mobilizações articulares, os alongamentos de cadeias musculares funcionais e o fortalecimento muscular ${ }^{6}$.

Em uma avaliação postural, são observados vários aspectos anatômicos estruturais, principalmente a estabilidade da criança na posição vertical inalterável. Logo, o paciente deve ser alinhado na postura ortostática pelo profissional, que delimita pontos de referência como descritores de marcação nas articulações do tornozelo, joelho, quadril, ombro, pescoço e cabeça para identificação dos desvios e anormalidades mais frequentes ${ }^{7}$.

Crianças com MMC apresentam diferentes níveis de comprometimento medular que são favoráveis para o desenvolvimento de um estudo mais específico, propiciando em particular, uma avaliação postural. Sabendo que essa patologia implica um atraso no desenvolvimento neuropsicomotor (DNPM) da criança, o presente estudo procura analisar: qual é a relação das alterações do alinhamento biomecânico com o nível de lesão funcional?

Considerando importante conhecer o perfil das crianças acometidas pela patologia, surgiu o interesse de procurar informações que apontassem para as alterações posturais, bem como seus respectivos níveis de acometimento, classificações e particularidades, a fim de identificar quais são as deformidades motoras existentes nos pacientes portadores de MMC. Deste modo, tornou-se indispensável no estudo o objetivo de realizar uma revisão sistemática acerca da avaliação postural de crianças com mielomeningocele.

\section{MATERIAL E MÉTODO}

O referido estudo se caracteriza em uma revisão, de categoria específica, com originalidade abrangente, estratégia de busca evidente, com método criterioso de avaliação, resumo quantitativo em frequência e resultados fundamentados em pesquisas clínicas.

Segundo Rother $^{8}$, o modo metódico presente numa revisão de estudos possui um valor original, pois além de utilizar os dados expostos na literatura como referência para investigação de determinado tema, esta modalidade de pesquisa possibilita uma importante apreciação de rigor metodológico, que é imprescindível para esclarecer a particularidade do contexto inserido na pesquisa.

A Biblioteca Virtual em Saúde (BVS) foi empregue para realizar a coleta das informações acerca da temática. Os artigos foram selecionados nas seguintes bases de dados: Medical Literature Analysis and Retrieval System Online (MEDLINE), Literatura Latino-Americana e do Caribe em Ciências da Saúde (LILACS), Scientific Electronic Library Online (SCIELO), US National Library of Medicine National Institutes of Health (PUBMED) e acervos de bibliotecas virtuais.

O estudo foi iniciado no mês de abril, sendo desenvolvido a partir da situação-problema indagada pela autora da pesquisa. Desse modo, o planejamento para esta revisão foi constituído por artigos ancorados ao setor de saúde e publicados entre os anos de 2007 e 2017.

Os descritores definidos para a pesquisa foram: mielomeningocele, disrafismo espinhal, postura, fisioterapia e crianças. Logo, o presente estudo compreende 
detalhadamente os aspectos elementares e exclusivos que ospacientes com espinha bífida aberta apresentam em seu perfil.

Foram obtidos como critérios de inclusão no estudo, artigos: completos, gratuitos e pagos, de revisões literárias e de procedimentos experimentais, estando em consenso com a temática determinada, nos idiomas português, inglês e alemão, organizados nas bases de dados. Critérios de exclusão foram estabelecidos para artigos incompletos, projetos em andamento, monografias, dissertações de mestrado, teses de doutorado, estudos de caso, com publicações anteriores ao ano de 2007 e contextos não condizentes com o tema em questão.

\section{RESULTADOS}

Foi localizada uma soma de 346 estudos com o uso dos descritores já mencionados, anteriormente. Foram excluídos 297 artigos científicos após inadequação e incoerência dos títulos. Permaneceram 49 artigos para leitura das sínteses. Posteriormente à interpretação dos resumos, 14 artigos foram separados para exploração dos critérios de inclusão e exclusão. Sete artigos foram eleitos para produzir esta revisão sistemática.

Para melhor compreensão dos resultados, foi construído um elemento gráfico em formato de organograma (Figura 2), que mostra detalhadamente a estrutura hierárquica da busca e coleta dos artigos envolvidos no presente estudo. À vista disso, as informações foram reunidas e organizadas segundo os graus de importância, em conformidade com a posição das variáveis.

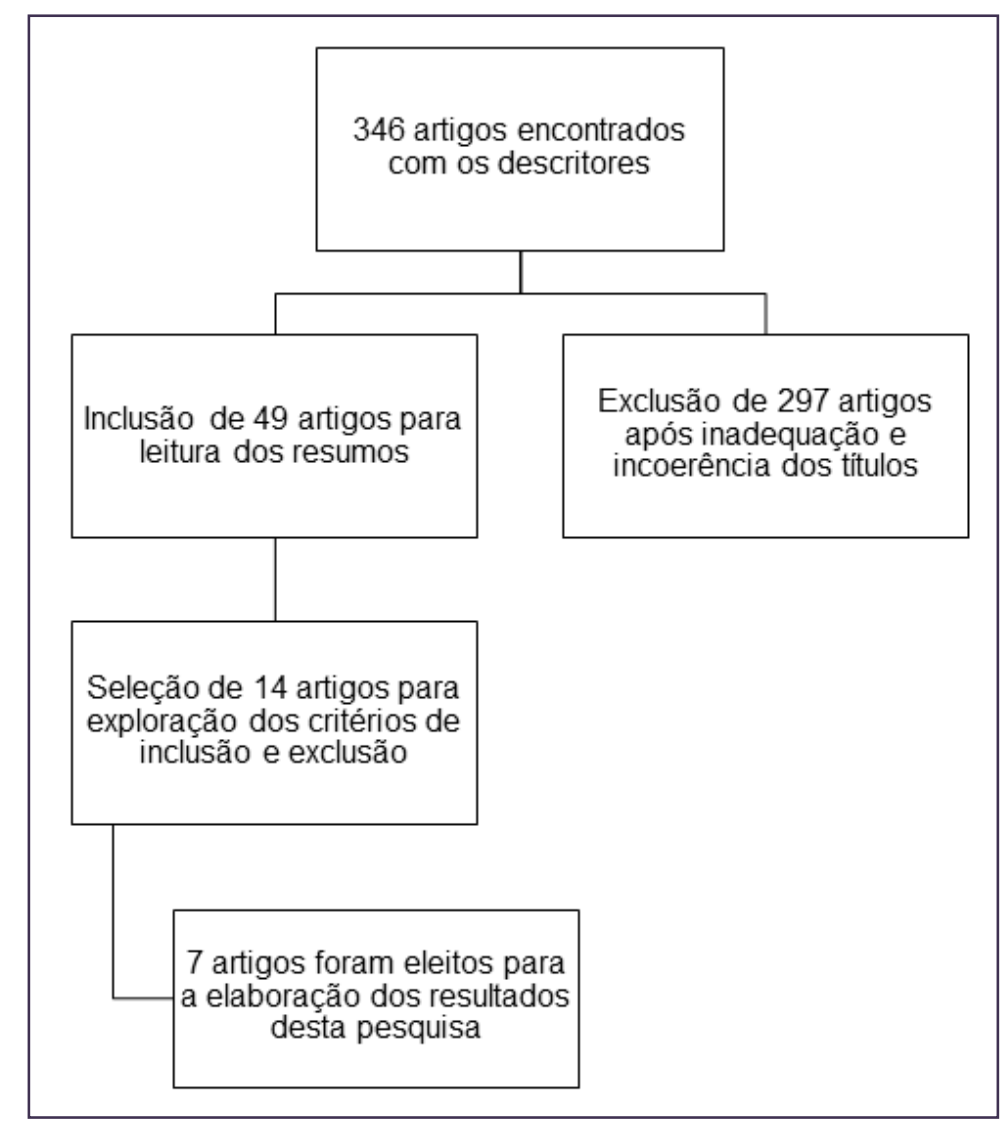

Figura 2. Elemento gráfico organizacional: organograma Fonte: Dados da pesquisa, 2017

Os artigos selecionados para compor essa divisão da pesquisa foram intitulados com conteúdos pertencentes à área de neuropediatria, visando compreender com facilidade o procedimento de avaliação postural em crianças com mielomeningocele.

Deste modo, para possibilitar um maior aproveitamento da leitura e entendimento dos resultados provenientes de cada elemento inserido, foi desenvolvida a Tabela 1, que esquematiza os dados descobertos nos demais artigos, outrora selecionados para elaboração desta revisão sistemática, especificando em ordem alfabética o autor e ano de publicação, o tipo de estudo utilizado, a metodologia aplicada e os resultados apurados.
Tabela 1. Relação dos artigos eleitos para elaboração da revisão

\begin{tabular}{|c|c|c|c|}
\hline $\begin{array}{l}\text { AUTOR } \\
\text { (ANO) }\end{array}$ & $\begin{array}{l}\text { TIPO DE } \\
\text { ESTUDO }\end{array}$ & METODOLOGIA & RESULTADOS \\
\hline $\begin{array}{l}\text { Biedermann } \\
\quad(2014)\end{array}$ & $\begin{array}{l}\text { Revisão da } \\
\text { literatura }\end{array}$ & $\begin{array}{l}\text { Levantamento de } \\
\text { informações com } \\
\text { registros } \\
\text { fotográficos acerca } \\
\text { do desalinhamento } \\
\text { corporal. }\end{array}$ & $\begin{array}{l}\text { As deformidades } \\
\text { detectadas foram } \\
\text { desvios posturais, } \\
\text { deslocamento de } \\
\text { quadril e pés } \\
\text { tortos. }\end{array}$ \\
\hline $\begin{array}{l}\text { Gaíva et } \\
\text { al.(2011) }\end{array}$ & $\begin{array}{l}\text { Descritivo, de } \\
\text { caráter } \\
\text { transversal, } \\
\text { com } \\
\text { abordagem } \\
\text { quantitativa }\end{array}$ & $\begin{array}{l}\text { Questionário reali- } \\
\text { zado com } 32 \text { crian- } \\
\text { ças que possuíam } \\
\text { mielomeningocele. }\end{array}$ & $\begin{array}{l}\text { Dentre os } \\
\text { problemas do } \\
\text { sistema motor, } 29 \\
(70 \%) \text { pacientes } \\
\text { envolvidos no } \\
\text { estudo } \\
\text { apresentaram pé } \\
\text { torto congênito. }\end{array}$ \\
\hline $\begin{array}{c}\text { Swaroop e } \\
\text { Dias } \\
\text { (2011) }\end{array}$ & $\begin{array}{l}\text { Revisão da } \\
\text { literatura }\end{array}$ & $\begin{array}{lr}\text { Por meio } & \text { de } \\
\text { registros } & \\
\text { fotográficos, } & \text { escla- } \\
\text { receram } & \text { os } \\
\text { desajustes } & \text { bio- } \\
\text { mecanicos } & \\
\text { presentes } & \text { nas } \\
\text { crianças } & \\
\text { acometidas. } & \end{array}$ & $\begin{array}{l}\text { Problemas } \\
\text { ortopédicos como } \\
\text { pés tortos. }\end{array}$ \\
\hline $\begin{array}{l}\text { Sá et al. } \\
\text { (2010) }\end{array}$ & $\begin{array}{l}\text { Descritivo, de } \\
\text { caráter } \\
\text { transversal, } \\
\text { com análise } \\
\text { institucional }\end{array}$ & $\begin{array}{l}\text { Análise de } 16 \text { pron- } \\
\text { tuários de } \\
\text { portadores de } \\
\text { mielomeningocele } \\
\text { em um hospital de } \\
\text { referência. }\end{array}$ & $\begin{array}{l}\text { Alterações ros } \\
\text { pés, } \\
\text { equinovaro } \\
\text { equinocavovaro. }\end{array}$ \\
\hline $\begin{array}{l}\text { Swaroop e } \\
\text { Dias } \\
(2009)\end{array}$ & $\begin{array}{l}\text { Revisão da } \\
\text { literatura }\end{array}$ & $\begin{array}{l}\text { As deformidades } \\
\text { ortopédicas foram } \\
\text { detectadas por in- } \\
\text { termédio de foto- } \\
\text { grafias. }\end{array}$ & $\begin{array}{l}\text { Contraturas, } \\
\text { subluxação do } \\
\text { quadril, problemas } \\
\text { nas articulações } \\
\text { dos membros } \\
\text { inferiores (Ml) } \\
\text { dor. }\end{array}$ \\
\hline $\begin{array}{c}\text { Braz et al. } \\
(2008)\end{array}$ & Intervencional & $\begin{array}{l}\text { Fotogrametria por } \\
\text { meio de um soft- } \\
\text { ware de avaliação } \\
\text { postural SAPO®. }\end{array}$ & $\begin{array}{l}\text { Foi constatado } \\
\text { que o software } \\
\text { SAPO } \AA \text { é uma } \\
\text { alternativa } \\
\text { fidedigna para } \\
\text { realizar medidas } \\
\text { angulares nos } \\
\text { segmentos } \\
\text { corporais. }\end{array}$ \\
\hline $\begin{array}{l}\text { Santos et al. } \\
\quad(2007)\end{array}$ & $\begin{array}{l}\text { Revisão da } \\
\text { literatura }\end{array}$ & $\begin{array}{l}\text { A partir de outros } \\
\text { estudos, discutiram } \\
\text { sobre as alterações } \\
\text { no alinhamento bio- } \\
\text { mecânico em dife- } \\
\text { rentes níveis de } \\
\text { lesão medular. }\end{array}$ & $\begin{array}{l}\text { Deformidades } \\
\text { ortopédicas como } \\
\text { hipercifose, } \\
\text { escoliose } \\
\text { alterações nos } \\
\text { quadris, joelhos e } \\
\text { pés. }\end{array}$ \\
\hline
\end{tabular}

*Fonte: Dados da pesquisa, 2017.

\section{DISCUSSÃO}

Os dados encontrados na presente pesquisa são característicos de uma população infantil com perfil de mielomeningocele e correspondem a bons resultados por meio do método de avaliação postural, que se fundamenta na inspeção minuciosa das limitações posturais resultantes de irregularidades no corpo.

Os resultados obtidos numa análise de postura, frequentemente, são de caráter vigente e possuem facilidade na aplicabilidade, já que são acessíveis e de custo econômico. Em vista disso, a maioria dos estudos apresentou o pé torto congênito como traço dominante nos pacientes com MMC, chegando também ao acordo de que as crianças com alterações na biomecânica corporal necessitam passar por um exame que identifique tais modificações, seguido de uma intervenção segura e satisfatória.

Em um estudo realizado por Sá et al. ${ }^{9} 16$ pacientes manifestaram alguns desvios que influenciaram diretamente na postura. As anomalias foram observadas através dos prontuários médicos que traziam informações referentes ao acometimento medular. Das crianças incluídas na pesquisa, duas apresentaram péssimo prognóstico para marcha devido ao comprometimento em nível torácico, onze contiveram 
complicações em região lombar e as outras três expuseram lesões de categoria sacral. $\mathrm{Na}$ avaliação das fichas, foi verificado que catorze $(93,3 \%)$ pacientes exteriorizaram desajustes no alinhamento biomecânico, sendo mais comum o pé torto congênito em condição equinovaro e equinocavovaro.

Seguindo as considerações presentes numa abordagem realizada pelos autores Gaíva et al. ${ }^{4}$, a aplicação de um questionário com 32 crianças diagnosticadas com espinha bífida aberta resultou que $70 \%$ dos pacientes entrevistados apresentaram pé torto congênito, cuja alteração provoca limitação na funcionalidade.

Concordando com o método aplicado em uma avaliação postural, a visualização dos segmentos corporais deve exprimir relação com o centro gravitacional. Dessa forma, as articulações de todo o corpo são analisadas e comparadas com o alinhamento ideal da postura, sendo assim, tudo aquilo que se desviar da normalidade padrão é considerado como desajuste.

As alterações no alinhamento são decorrentes das variações que afetam o aspecto motor em pacientes acometidos pela MMC. Para Santos et al. ${ }^{10}$, apesar de seu estudo não efetuar a prática de uma análise postural, através de um levantamento bibliográfico, reconheceram que as deformidades mais habituais encontradas nessas crianças são de traços ortopédicos, que se estendem por várias porções do corpo, tais como desvios nas curvas da coluna vertebral com hipercifose e escoliose, e também modificações de um posicionamento estável nas articulações, principalmente nos membros inferiores (MMII).

Na direção das mudanças posturais, Swaroop e Dias ${ }^{11}$ apresentaram fundamento em outros estudos acerca dos desalinhamentos estruturais. Também constataram que as desvantagens existentes interferem o desenvolvimento motor da criança e se relacionam com o nível de lesão medular, comprometendo continuamente o sistema neurológico. Ainda explanaram, através de registros fotográficos, que as alterações mais frequentes de serem detectadas são as deformidades ortopédicas, com realce para os pés tortos mais uma vez.

Os mesmos autores, Swaroop e Dias ${ }^{12}$, em outra publicação determinaram para as mensurações acerca do desenvolvimento postural que as crianças com MMC tendem a desenvolver uma variedade de problemas ortopédicos desde o nascimento. Também por meio de fotografias, observaram que as disformidades que acometem a condição locomotora, bem como as alterações musculoesqueléticas estão unidas à fraqueza muscular. Outras dificuldades foram evidenciadas como a presença das contraturas musculares que influenciam diretamente a manutenção da postura, a subluxação de quadril e dor, em alguns casos.

Com relação ao uso das ferramentas necessárias para executar uma avaliação postural, há uma clara convergência a respeito da opinião dos autores pesquisados. Aqueles que se submeteram a realizar a exploração utilizaram o registro fotográfico de modo leal como principal recurso para obtenção de dados.

Segundo Biedermann ${ }^{13}$ a fotografia quando utilizada como ferramenta para registrar o desalinhamento corporal acerca de detectar as disfunções e desvios posturais, é um meio eficaz que exige determinado rigor para observar as deformidades particulares dos diferentes níveis de comprometimento medular.

Considerando as informações referidas anteriormente e no que diz respeito à confiabilidade para uso das fotos em uma avaliação postural, Braz et al. ${ }^{14}$ optaram associar a fotogrametria com outro recurso. Nesse caso, eles concordaram em experimentar o software $\mathrm{SAPO}{ }^{\circledR}$, programa simples que demonstrou satisfatório desempenho ao identificar a mensuração angular dos segmentos do corpo, de modo fidedigno e seguro.

\section{CONCLUSÃO}

Com base nos dados obtidos foi possível constatar que a tipologia da pesquisa como revisão sistemática gerou resultados satisfatórios relacionados com a avaliação postural de crianças com mielomeningocele.

Tais fatos evidenciaram que os impactos físicos existentes nos portadores infantis correspondem a problemas na estrutura dos segmentos anatômicos, de modo mais relevante o pé torto congênito.

Assim, os efeitos alcançados tendem a contribuir de maneira significativa para o meio acadêmico e para os demais profissionais da área de saúde, em especial ao fisioterapeuta, que além de identificar os desníveis existentes no alinhamento corporal, também é capaz de intervir recuperando a mobilidade funcional deficitária dos pacientes.

Nesse sentido, a vantagem desta pesquisa em relação aos demais explorados é o interesse no aprofundamento da temática, visto que a academia científica padece de uma ponderosa escassez acerca do tema.

\section{REFERÊNCIAS}

1. Collange LA, Franco RC, Esteves RN, Zanon-Collange N. Desempenho funcional de crianças com mielomeningocele. Fisioterapia e Pesquisa. 2008;15(1):58-63.

2. Santos SA, Souza MIAW, Calasans MTA. Perfil de crianças e adolescentes com mielomeningocele. Rev Enferm UFPE. 2014; 8(6):1642-8.

3. Kliegman RM, Stanton BF, Schor NF, Geme JW, Behrman RE. Nelson, tratado de pediatria. $19^{\mathrm{a}}$ ed. Rio de Janeiro: Elsevier; 2014.

4. Gaíva MAM, Corrêa ER, Espírito Santo EAR. Estudo das variáveis materno-infantis na espinha bífida. Rev Bras Cresc e Desenv Hum. 2011; 21(1):99-110.

5. Rocco FM, Saito ET, Fernandes AC. Perfil dos pacientes com mielomeningocele da Associação de Assistência à Criança Deficiente (AACD) em São Paulo - SP, Brasil. Acta Fisiátrica. 2007; 14(3):130-3.

6. Kopczynski MC. Fisioterapia em neurologia. Barueri: Manole; 2012.

7. Oatis CA. Cinesiologia: a mecânica e a patomecânica do movimento humano. 2. ed. Barueri: Manole; 2014.

8. Rother ET. Revisão sistemática $x$ revisão narrativa. Acta paul enferm. 2007; 20(2):v-vi.

9. Sá MRCO, Abelheira M, Sohler L, Puccioni M. Perfil de crianças com mielomeningocele em hospital de referência - Rio de Janeiro. Rev Bras Neurol. 2010; 46(4):7-11.

10. Santos CMT, Pereira CU, Santos EAS, Monteiro JTS. Reabilitação na mielomeningocele. Rev Bras Med. 2007; 64(11):518-20.

11. Swaroop VT, Dias L. Orthopaedic management of spina bifida-part II: foot and ankle deformities. J Child Orthop. 2011; 5(6):403-14.

12. Swaroop VT, Dias L. Orthopedic management of spina bifida-part I: hip, knee, and rotational deformities. J Child Orthop. 2009; 3(6):441-9.

13. Biedermann R. Orthopädisches management der spina bifida. Der Orthopäde, 2014.

14. Braz RG, Goes FPDC, Carvalho GA. Confiabilidade e validade de medidas angulares por meio do software para avaliação postural. Fisioter Mov. 2008; 21(3):117-26. 


\section{CONFLITO DE INTERESSES}

Os autores declaram não haver conflitos de interesse.

\section{AUTOR PARA CORRESPONDÊNCIA}

Thaynara do Monte Mélo

mmelothaynara@gmail.com

Submetido em 05/12/2017

Aceito em 09/01/2018 